\title{
First-Principles Calculations of Structural, Electronic, Optical, and Thermodynamic Properties of CdS, CdTe and Their Ternary Alloys $\mathrm{CdS}_{1-x} \mathrm{Te}_{x}(0.0 \leq x \leq 1.0)$
}

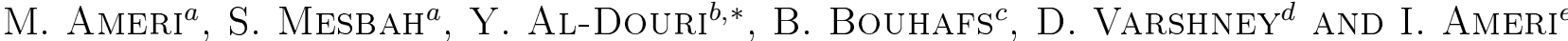 \\ ${ }^{a}$ Laboratory Physico-Chemistry of Advanced Materials, University of Djillali Liabès \\ P.O.B. 89, Sidi-Bel-Abbès, 22000, Algeria \\ ${ }^{b}$ Institute of Nano Electronic Engineering, University Malaysia Perlis, 01000 Kangar, Perlis, Malaysia \\ ${ }^{c}$ Laboratoire de Modélisation et Simulation en Sciences des Matériaux, Université Djilali Liabès \\ Sidi-Bel-Abbès, 22000, Algeria \\ ${ }^{d}$ Materials Science Laboratory, School of Physics, Vigyan Bhavan, Devi Ahilya University \\ Khandwa Road Campus, Indore 452001, India \\ ${ }^{e}$ University of Djillali Liabès, Faculty of Exact Sciences, Department of Physics \\ P.O.B. 089, Sidi-Bel-Abbès, 22000, Algeria \\ (Received February 27, 2013; revised version October 31, 2013; in final form December 31, 2013) \\ Using first-principle method, we investigate the structural, electronic, optical, and thermodynamic properties \\ of the $\mathrm{CdS}_{1-x} \mathrm{Te}_{x}$ semiconductor alloys using generalized gradient approximation for the exchange-correlation \\ potential calculation. The ground state properties are determined for the bulk materials (CdS and $\mathrm{CdTe}$ ) in \\ cubic phase. Quantities such as the lattice constants and bulk modulus of interest are calculated. Detailed \\ comparisons are made with published experimental and theoretical data and show generally good agreement. \\ The calculated lattice constants scale linearly with composition (Vegard's law). The microscopic origins of the \\ bowing parameter were explained using the contributions from volume deformation, charge transfer and structural \\ relaxation approach. The refractive index and optical dielectric constant for the alloys of interest were calculated \\ by using different models. In addition, the thermodynamic stability of the alloys was investigated by calculating \\ the critical temperatures of alloys.
}

DOI: $10.12693 /$ APhysPolA.125.1110

PACS: 31.15.E-; 63.20.dk; 04.25.-g; 78.66.Hf; 73.61.At

\section{Introduction}

The Cd-based binary semiconductors are very important, owing to their direct and rather large gap. These materials are the cornerstones of technological activity. One possible reason is to produce novel materials such as blue-green laser diode based on these compounds. Recently, the cadmium chalcogenides $\mathrm{CdX}(\mathrm{X}=\mathrm{S}$, Se, and $\mathrm{Te}$ ) have attracted special attention by various researchers $[1,2]$. CdX semiconductor compounds also noticeably are used to make thin film solar cells, photodetectors and other optoelectronic devices [3]. Up today, many theoretical methods are available such as $a b i n i$ tio method, density function theory, molecular dynamics, etc. To investigate the structural [3-5], electronic [6-8], thermodynamic $[10,11]$ and optical properties for cadmium based chalcogenides [12-14].

Recently, there have been numerous calculations [15] of the structural, electronic, and optical properties of these compounds using full-potential linearized augmented plane wave (FP-LAPW) method coupled with the local density approximation (LDA) [16] of the ex-

*corresponding author; e-mail: yaldouri@yahoo.com change correlation energy to calculate electronic and optical properties, respectively. To our knowledge there are few reports that had used the full potential calculation FP-LAPW to calculate the electronic, structural, and optical properties for these chalcogenides based compounds.

Concerning the $\mathrm{CdS}_{1-x} \mathrm{Te}_{x}$ alloy, to the best of our knowledge, many experimental values of $E_{\mathrm{g}}$, optical and structural characterization of thin film $\mathrm{CdS}_{1-x} \mathrm{Te}_{x}$ thin films for solar cell applications [17-19], generally studying a small number of samples of different concentration $x$, but few theoretical investigations [20] have been appeared in the literature. However, these results represented a large amount of data, with the potential to provide a more reliable trend in $E_{\mathrm{g}}$.

In the present work, we thus aimed to combine $\mathrm{CdX}$ compounds having different structural and electronic properties in order to obtain new material, $\mathrm{CdS}_{1-x} \mathrm{Te}_{x}$ ternary alloy. The present work thus explores the detailed studies of the structural, electronic, optical, and thermodynamic properties of $\mathrm{CdS}_{1-x} \mathrm{Te}_{x}$ ternary alloy by using a full-potential linear muffin-tin orbital (FP-LMTO) method. The physical origins of bowing parameters are calculated following the approach of Zunger and co-workers [21].

The paper is divided into three parts. In Sect. 2, we briefly describe the computational techniques used in this 
study. The theoretical results and discussion concerning the structural, electronic, optical, and thermodynamic properties are presented in Sect. 3. Finally, in Sect. 4 we summarize the main conclusions of our work.

\section{Computational details}

The calculations here were carried out using the $a b$ initio FP-LMTO method as implemented in the Lmtart code [22-24]. The exchange and correlation potential was calculated using the gradient generalized approximation (GGA) [25]. This is an improved method compared to previous (LMTO) methods. The FP-LMTO method treats muffin-tin spheres and interstitial regions on the same footing, leading to improvements in the precision of the eigenvalues. At the same time, the FP-LMTO method, in which the space is divided into interstitial regions (IR) and non-overlapping muffin-tin spheres (MTS) surrounding the atomic sites, uses a more complete basis than its predecessors.

In the IR regions, the basis functions are represented by the Fourier series. Inside the MTS spheres, the basis functions are represented in terms of numerical solutions of the radial Schrödinger equation for the spherical part of the potential multiplied by spherical harmonics. The charge density and the potential are represented inside the MTS by spherical harmonics up to $l_{\max }=6$. The integrals over the Brillouin zone are performed up to 55 special $k$-points for binary compounds and 27 special $k$-points for the alloys in the irreducible Brillouin zone (IBZ), using the Blöchl modified tetrahedron method [26].

\section{TABLE I}

The plane wave number PW, energy cut-off (in Ry) and muffin-tin radius (RMT) (in arb.u.) used in calculation for binary $\mathrm{CdS}, \mathrm{CdTe}$ and their alloys.

\begin{tabular}{c|c|c|c}
\hline \hline$x$ & PW & $E_{\text {cut total [Ry] }}$ & RMT (arb.u.) \\
\hline 0 & 12050 & 157.950 & Cd 2.50 \\
& & & S 2.40 \\
0.25 & 65266 & 178.840 & Cd 2.44 \\
& & & S 2.64 \\
& & & Te 2.64 \\
0.5 & \multirow{2}{*}{65266} & \multirow{2}{*}{168.441} & Cd 2.515 \\
& & & S 2.725 \\
& & & Te 2.725 \\
0.75 & 65266 & 160.287 & Cd 2.578 \\
& & & S 2.793 \\
& & & Te 2.793 \\
& \multirow{2}{*}{12050} & \multirow{2}{*}{125.786} & Cd 2.634 \\
& & & Te 2.854
\end{tabular}

The self-consistent calculations are considered to be converged when the total energy of the system is stable within $10^{-5} \mathrm{Ry}$. In order to avoid the overlap of atomic spheres the MTS radius for each atomic position is taken to be different for each case. Both the plane waves cut-off are varied to ensure the total energy convergence. The values of the sphere radii (MTS) and number of plane waves (NPLW) used in our calculation are summarized in Table I.

\section{Results and discussion}

\subsection{Structural properties}

To investigate the structural properties of $\mathrm{CdS}$ and CdTe compounds and their alloys in the cubic structure, we have started FP-LMTO calculation with the zinc-blende structure and let the calculation forces to move the atoms to their equilibrium positions (is shown in Fig. 1). We have chosen the basic cubic cell as the unit cell.

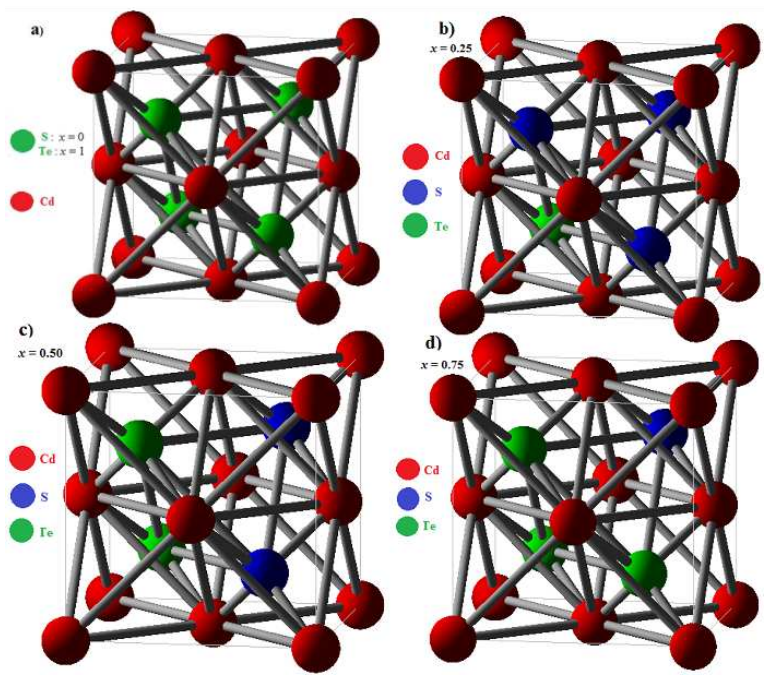

Fig. 1. Cubic crystal structure of $\mathrm{CdS}_{1-x} \mathrm{Te}_{x}$ alloy for $x=0$ and 1 (a), 0.25 (b), 0.50 (c), 0.75 (d).

In the unit cell there are four $\mathrm{C}$ anions, three $\mathrm{A}$ and one $\mathrm{B}$, two $\mathrm{A}$ and two $\mathrm{B}$, and one $\mathrm{A}$ and three $\mathrm{B}$ cations for $x=0.25,0.50$ and 0.75 , respectively (as shown in Fig. 1). For the considered structures, we perform the structural optimization by calculating the total energies for different volumes around the equilibrium cell volume $V_{0}$ of the binary $\mathrm{CdS}$ and CdTe compounds and their alloy.

The calculated total energies are fitted to the Murnaghan equation of state [27] to determine the ground state properties such as the equilibrium lattice constant $a$, and the bulk modulus $B$ (as shown in Fig. 2).

The equilibrium structural properties such as the lattice constants and bulk modulus were obtained for both binary compounds and their alloys, the results are given in Table II. Considering the general trend that GGA usually overestimates the lattice parameters [28], our GGA results of binary compounds are in good agreement with the experimental and other calculated values. 
Calculated lattice parameter $(a)$ and bulk modulus $(B)$ for cadmium chalcogenides and their alloys.

\begin{tabular}{c|c|c|c|c|c|c}
\hline \hline \multirow{2}{*}{$x$} & \multicolumn{3}{|c|}{ Lattice parameter $a[\AA]$} & \multicolumn{2}{c}{ Bulk modulus $B$ [GPa] } \\
\cline { 2 - 6 } & Our work & Exp. & Other cal. & Our work & Exp. & Other cal. \\
\hline \multicolumn{7}{c}{$\mathrm{CdS}_{1-x} \mathrm{Te}_{x}$} \\
\hline 0 & 5.986 & $5.82^{a}$ & $5.896^{b}, 5.94^{c}, 5.81^{d}, 5.81^{e}, 5.95^{f}$ & 50.8 & $62^{a}$ & $72.42^{d}, 64.63^{e}, 56.84^{f}$ \\
0.25 & 6.215 & & $6.147^{f}$ & 43.3 & & $46.61^{f}$ \\
0.5 & 6.404 & & $6.33^{c}, 6.325^{f}$ & 38.2 & & $41.6^{c}, 41.42^{f}$ \\
0.75 & 6.565 & & $6.481^{f}$ & 34.9 & & $37.26^{f}$ \\
1 & 6.708 & $6.48^{a}$ & $6.626^{b}, 6.48^{d}, 6.48^{e}, 6.631^{f}$ & 33.2 & $44.5^{a}$ & $44.5^{c}, 48.94^{d}, 41.76^{e}, 33.78^{f}$ \\
\hline
\end{tabular}

${ }^{a}$ Ref. [28], ${ }^{b}$ Ref. [30], ${ }^{c}$ Ref. [31], ${ }^{d}$ Ref. [19], ${ }^{e}$ Ref. [32], ${ }^{f}$ Ref. [33].

Energy gap $E_{\mathrm{g}}$ of chalcogenides and their alloys.

TABLE III

\begin{tabular}{c|c|c|c|c}
\hline \hline \multirow{2}{*}{$x$} & \multicolumn{3}{|c|}{ Energy gap [eV] $(\Gamma-\Gamma)$} & Energy gap [eV] $(\Gamma-X)$ \\
\cline { 2 - 4 } & Our work & Exp. & Other cal. & Our work \\
\hline \multicolumn{3}{|c}{$\mathrm{CdS}_{1-x} \mathrm{Te}_{x}$} \\
\hline 0 & 1.006 & $2.55^{a}$ & $1.11^{b}, 1.45^{c}, 0.93^{d}, 1.22^{d}, 0.999^{e}, 1.8^{e}$ & 4.186 \\
0.25 & 0.879 & & $0.873^{e}, 1.59^{e}$ & 3.142 \\
0.5 & 0.715 & & $0.750^{e}, 1.39^{e}$ & 2.873 \\
0.75 & 0.480 & & $0.657^{e}, 1.27^{e}$ & 2.575 \\
1 & 0.429 & $1.6^{a}$ & $0.62^{a}, 1.88^{c}, 0.60^{d}, 1.32^{d}, 0.588^{e}, 1.087^{e}$ & 2.636 \\
\hline
\end{tabular}

${ }^{a}$ Ref. [28], ${ }^{b}$ Ref. [30], ${ }^{c}$ Ref. [19], ${ }^{d}$ Ref. [32], ${ }^{e}$ Ref. [33].

Figure 3 shows the variation of the calculated equilibrium lattice constants and the bulk modulus versus concentration $x$ for $\mathrm{CdS}_{1-x} \mathrm{Te}_{x}$ alloy. Our calculated lattice parameters at different compositions of $\mathrm{CdS}_{1-x} \mathrm{Te}_{x}$ alloy were found to vary almost linearly following Vegard's law [29] with a marginal upward bowing parameter equal to $-0.22857 \AA$. The physical origin of this marginal bowing parameter should be mainly due to the weak mismatches of the lattice constants of $\mathrm{CdS}$ and CdTe compounds.

The composition dependence of bulk modulus is compared with the results predicted by linear concentration dependence (LCD) (Fig. 4). A significant deviation of the bulk modulus from the linear concentration dependence with downward bowing parameter equal to $15.314 \mathrm{GPa}$ is noticed. We note that the bulk modulus decreases as the concentration $(x)$ increases. Hence, we conclude that CdTe is more compressible compared to CdS binary compound.

\subsection{Electronic properties}

The important features of the band structure (direct $\Gamma-\Gamma$ and indirect $\Gamma-X$ band gaps) are given in Table III (the band structures of the ternary $\mathrm{CdS}_{1-x} \mathrm{Te}_{x}$ is represented by Fig. 5). It is clearly seen that the band gaps as a whole are underestimated as comparison with the experimental results. This underestimation of the band gaps is mainly due to the fact that the GGA does not take into account the quasi-particle self-energy correctly [36] which make them insufficient flexible to accurately reproduce both exchange and correlation energy and its charge derivative. We also mention that it is far to say that the experimental data are well reproduced by the calculation.

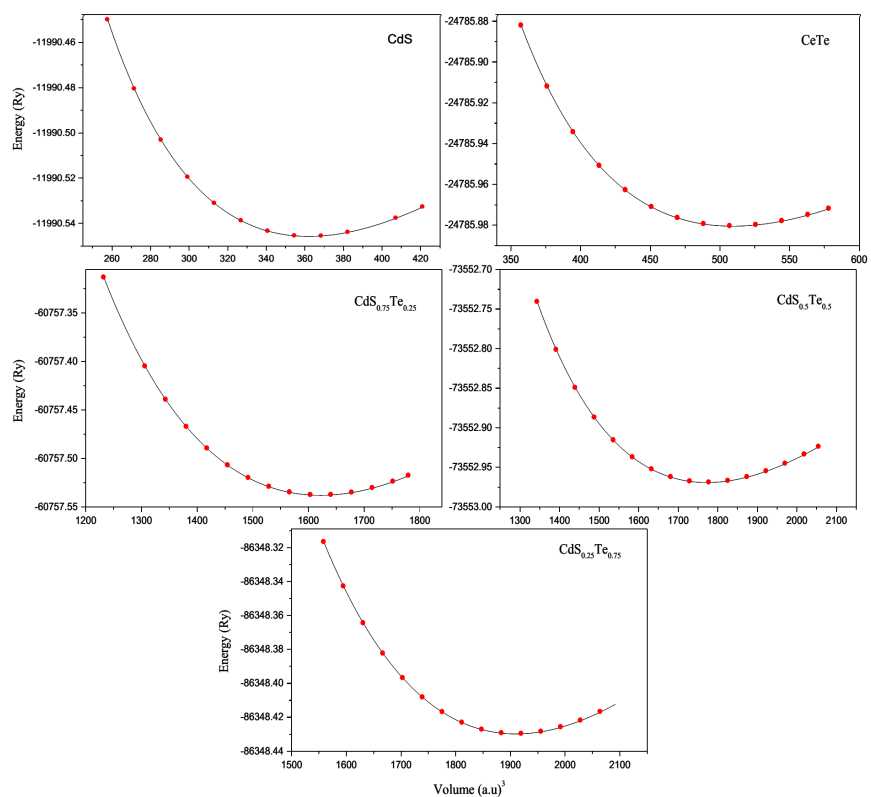

Fig. 2. Total energy as a function of the volume with GGA calculation for $\mathrm{CdS}_{1-x} \mathrm{Te}_{x}$ alloy for $x=0,0.25$, $0.50,0.75$ and 1 .

One reason for this difference is that in our calculations we have assumed the crystal to be at $T=0 \mathrm{~K}$ and thus do not include contributions from lattice vibrations that are present at room temperature measurements.

Figure 6 presents the variation of the direct and indirect band gap energies as functions of the composition $(x)$ 


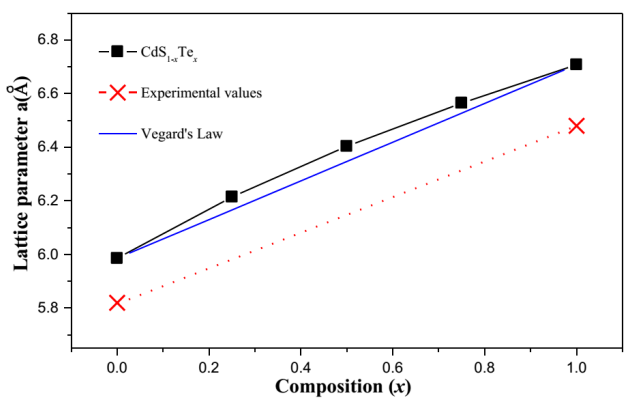

Fig. 3. Composition dependence of the calculated lattice constants (solid squares) of $\mathrm{CdS}_{1-x} \mathrm{Te}_{x}$ alloy compared with Vegard's prediction (dotted line).

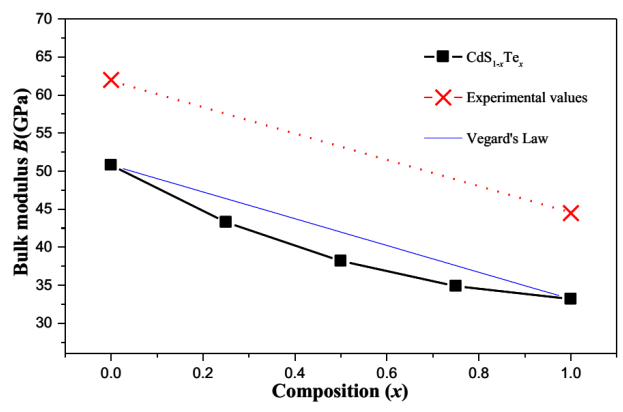

Fig. 4. Variation of the calculated bulk modulus versus $x$ concentration of $\mathrm{CdS}_{1-x} \mathrm{Te}_{x}$ alloy (solid line) compared with LCD prediction (dotted line).
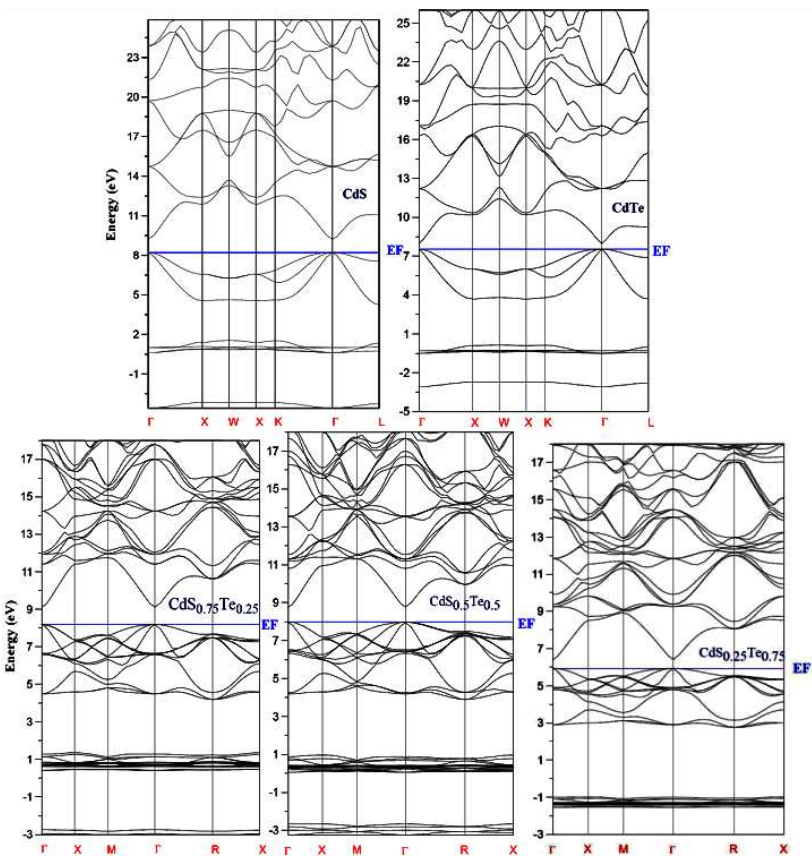

Fig. 5. Band structure of ternary alloy $\mathrm{CdS}_{1-x} \mathrm{Te}_{1-x}$ for $x=0,0.25,0.50,0.75$ and 1 .

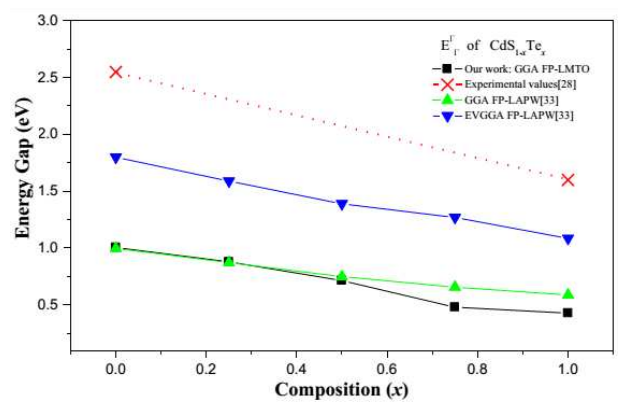

Fig. 6. Energy band gap of $\mathrm{CdS}_{1-x} \mathrm{Te}_{x}$ alloy as a function of composition $x$.

for the ternary alloys. We note that direct $(\Gamma-\Gamma)$ and indirect $(\Gamma-X)$ band gaps are not intersecting as the computed band structures of the alloys indicate a direct band gap at various concentrations. The calculated band gap versus concentrations was fitted by a polynomial equation. The results are summarized as follows with another found experimentally $E_{\mathrm{g}}=1.54-1.06 x+1.88 x^{2}[17]$ :

$$
\begin{aligned}
& E_{\Gamma-\Gamma}=1.02-0.71 x+0.09 x^{2}, \\
& E_{\Gamma-X}=4.12-3.95 x+2.49 x^{2} .
\end{aligned}
$$

To understand the physical origins of the bowing parameter in $\mathrm{CdS}_{1-x} \mathrm{Te}_{x}$ alloy, we follow the procedure of Bernard and Zunger [21] of decomposing it into three contributions. Since the compositional effect on the bowing parameter is considered to be small, the band gap bowing equations of Bernard and Zunger have been defined only by the contributions of the volume deformation $\left(b_{\mathrm{VD}}\right)$, charge transfer $\left(b_{\mathrm{CE}}\right)$ and the structural relaxation $\left(b_{\mathrm{SR}}\right)$ of the alloys as follows:

$$
b=b_{\mathrm{VD}}+b_{\mathrm{CE}}+b_{\mathrm{SR}} \text {. }
$$

The calculated bowing parameter contributions of the direct band gap are presented in Table IV. The total bowing parameter for $\mathrm{CdS}_{1-x} \mathrm{Te}_{x}$ alloy has been found to be very small. The small value of $b_{\mathrm{VD}}$ is related to the weak mismatch of the lattice parameters of $\mathrm{CdX}$ compounds and that of $b_{\mathrm{CE}}$ is due to the weak electronegativity [37] difference between $\mathrm{CdS}$ and $\mathrm{CdTe}$ atoms. The small contribution of the structural relaxation to the bowing parameter is due to that our calculations are for ordered structure.

\subsection{Effective mass calculation}

The knowledge of the electron and hole effective mass values is indispensable for the understanding of transport phenomena, exciton effects and electron-hole in semiconductors. Therefore, it would be of much interest to determine the electron and hole effective mass values for the alloys for various doping concentration. We have computed the electron effective mass at the conduction band minima (CBM) and hole effective mass at the valence band maxima (VBM) for the $\mathrm{CdS}_{1-x} \mathrm{Te}_{x}$ alloy. The electron and hole effective masses values are then obtained from the curvature of the energy band near the $\Gamma$-point at the CBM and VBM for all concentration. At the $\Gamma$-point 


\section{TABLE IV}

Decomposition of optical bowing into volume deformation (VD), charge exchange (CE), and structural relaxation (SR) contributions (all values are in $\mathrm{eV}$ ).

\begin{tabular}{c|c|c}
\hline \hline$x$ & Our work & Other cal. \\
\hline 0.25 & & \\
$b_{\mathrm{VD}}$ & -2.825 & \\
$b_{\mathrm{CT}}$ & 0.884 & \\
$b_{\mathrm{SR}}$ & 0.309 & \\
$b$ & -1.632 & \\
0.5 & & \\
$b_{\mathrm{VD}}$ & -0.168 & $-0.126^{a},-0.046^{a}$ \\
$b_{\mathrm{CT}}$ & -0.0925 & $0.179^{a}, 0.032^{a}$ \\
$b_{\mathrm{SR}}$ & 0.272 & $0.120^{a}, 0.228^{a}$ \\
$b$ & 0.011 & $0.173^{a}, 0.214^{a}$ \\
0.75 & & \\
$b_{\mathrm{VD}}$ & 2.075 & \\
$b_{\mathrm{CT}}$ & -0.865 & \\
$b_{\mathrm{SR}}$ & 0.823 & \\
$b$ & 2.033 & \\
\hline \multicolumn{2}{c}{$\operatorname{Ref.}[29]}$.
\end{tabular}

the $s$-like conduction band effective mass can be obtained through a simple parabolic fit using the definition of the effective mass as the second derivative of the energy band with respect to the wave vector, $k$, via

$$
m^{*} / m_{0}=-\left(\hbar^{2} / m_{0}\right) \frac{1}{\mathrm{~d}^{2} E / \mathrm{d} k^{2}} .
$$

Here, $m^{*}$ is the conduction electron effective mass and $m_{0}$ is the free electron mass. We can then calculate the curvature of the valence band maximum with the following assumptions: if the spin-orbit interactions were neglected, the top of the valence band would have a parabolic behavior; this implies that the highest valence bands are parabolic in the vicinity of the $\Gamma$ point. In this work, CdS, CdTe and $\mathrm{CdS}_{1-x} \mathrm{Te}_{x}$ alloys satisfy this parabolic condition of the valence band maximum at the $\Gamma$-point. Within this approach, and by using the appropriate expression of Eq. (4) (using a plus sign instead of the minus sign in the prefactor), we have computed the effective masses of the heavy and light holes at the $\Gamma$ point. Table $\mathrm{V}$ shows the calculated effective masses for the binary compounds, CdS and CdTe as well for $\mathrm{CdS}_{1-x} \mathrm{Te}_{x}$ alloy. This table also includes theoretical and experimental values that were reported in literature, for comparison. The calculated effective masses show that the holes are much heavier than the electrons, so carrier transport in $\mathrm{CdS}_{1-x} \mathrm{Te}_{x}$ should be dominated by electrons. Our results concerning the electrons, heavy holes and light hole effective mass are shown in Fig. 7.

\subsection{Linear optical properties}

Quite generally, the basic optical properties of semiconductors result from the electronic excitation in crystals when an electromagnetic wave is incident on them. The calculation of the optical properties of the solids is beset

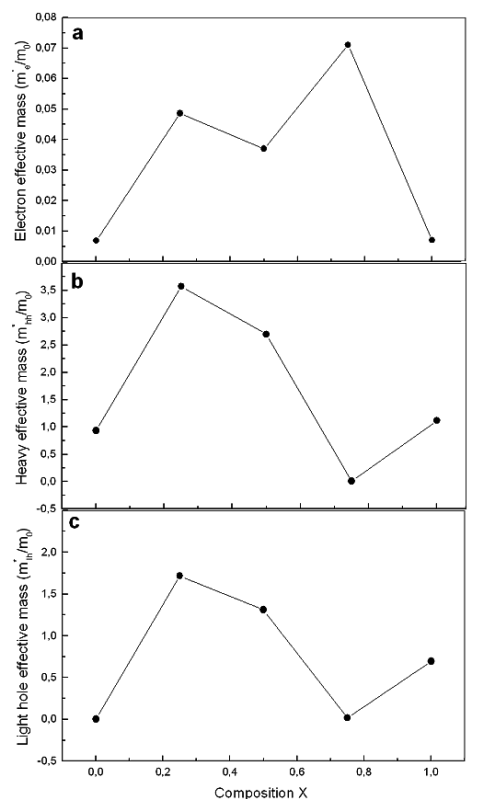

Fig. 7. (a) Electron effective mass at point $\Gamma$. (b) Heavy hole effective mass (in units of free electron mass $\left.m_{0}\right)$. (c) Light hole effective mass at point $\Gamma$ of $\mathrm{CdS}_{1-x} \mathrm{Te}_{x}$ alloy as a function of composition $x$.

TABLE V

Electron $\left(m_{\mathrm{e}}^{*}\right)$, heavy hole $\left(m_{\mathrm{hh}}^{*}\right)$ and light hole $\left(m_{\mathrm{lh}}^{*}\right)$ effective masses (in units of free electron mass $m$ ).

\begin{tabular}{c|c|c|c}
\hline \hline$x$ & $m_{\mathrm{e}}^{*}$ & $m_{\mathrm{hh}}^{*}$ & $m_{\mathrm{lh}}^{*}$ \\
\hline \multicolumn{4}{|c}{$\mathrm{CdS}_{1-x} \mathrm{Te}_{x}$} \\
\hline 0 & 0.00684 & 0.9363 & 0.00402 \\
0.25 & 0.0486 & 3.5741 & 1.719 \\
0.5 & 0.037 & 2.6963 & 1.311 \\
0.75 & 0.071 & 0.00654 & 0.0187 \\
1 & 0.00699 & 1.1178 & 0.695357
\end{tabular}

with numerous problems. The knowledge of the dielectric functions $\varepsilon(\omega)=\varepsilon_{1}(\omega)+\mathrm{i} \varepsilon_{2}(\omega)$ allows describing the optical properties of the medium at all phonon energies. Calculations of the dielectric function involve the energy eigenvalues and the electron wave functions. These are the natural output of the $a b$ initio band structure calculation which is usually performed under GGA [38, 39]. We have calculated the frequency dependent imaginary dielectric function and real dielectric function. The effects of using $K$ points in the Brillouin zone (BZ) have already been discussed in the earlier work [40].

The knowledge of both real and imaginary parts of the dielectric function allows the calculation of important optical functions. In this paper, we also present and analyze the refractive index $\varepsilon(\omega)$ given by

$$
n(\omega)=\left[\frac{\varepsilon_{1}(\omega)}{2}+\frac{\sqrt{\varepsilon_{1}^{2}(\omega)+\varepsilon_{2}^{2}(\omega)}}{2}\right]^{1 / 2} .
$$

At low frequency $(\omega=0)$, we obtain the following rela- 
tion:

$$
n(0)=\varepsilon^{\frac{1}{2}}(0) .
$$

The refractive index $n$ of semiconducting materials is very important in determining the optical and electric properties of the crystal. Knowledge of $n$ is essential in the design of heterostructure lasers, and optoelectronic devices. In the present study, the refractive index has been obtained using different models that are related to the fundamental energy band gap.

1. The Moss formula [41] is based on atomic model

$$
E_{\mathrm{g}} n^{4}=k \text {. }
$$

Here, $E_{\mathrm{g}}$ is energy gap, $n$ is the refractive index, and $k$ is the attenuation index, also is called the extinction coefficient.

2. The Ravindra et al. [42] relation is

$$
n=\alpha+\beta E_{\mathrm{g}},
$$

where $\alpha$ and $\beta$ are constants.

3. Herve and Vandamme's empirical relation [43]:

$$
n=\sqrt{1+\left(\frac{A}{E_{\mathrm{g}}+B}\right)^{2}},
$$

where $A$ and $B$ are constants.

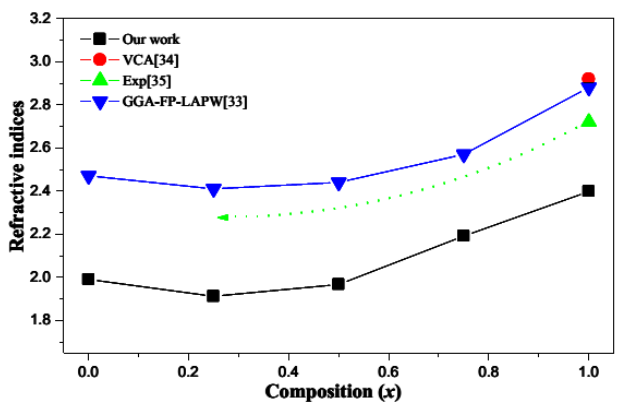

Fig. 8. Variation of the calculated refractive index versus $x$ concentration of $\mathrm{CdS}_{1-x} \mathrm{Te}_{x}$ alloy.

Refractive indices of $\mathrm{CdS}_{1-x} \mathrm{Te}_{x}$ for different compositions $x$.

TABLE VI

\begin{tabular}{c|c|c|c|c|c|c|c|c|c}
\hline \hline \multirow{2}{*}{$x$} & \multicolumn{5}{c|}{ This work } & \multicolumn{5}{c}{ Other cal. } \\
\cline { 2 - 10 } & FP-LMTO & Relation (7) & Relation (8) & Relation (9) & FP-LAPW & Relation (7) & Relation (8) & Relation (9) & For $x=1$ \\
\hline \multicolumn{7}{c}{ CdS $_{1-x} \mathrm{Te}_{x}$} \\
0.25 & 1.991 & 3.218 & 3.460 & 3.244 & $2.47^{a}$ & $3.224^{a}$ & $3.446^{a}$ & $3.249^{a}$ & \\
0.5 & 1.913 & 3.329 & 3.539 & 3.331 & $2.41^{a}$ & $3.335^{a}$ & $3.542^{a}$ & $3.336^{a}$ & \\
0.75 & 1.968 & 3.505 & 3.640 & 3.452 & $2.44^{a}$ & $3.464^{a}$ & $3.61^{a}$ & $3.426^{a}$ & \\
1 & 2.192 & 3.872 & 3.786 & 3.645 & $2.57^{a}$ & $3.58^{a}$ & $3.676^{a}$ & $3.498^{a}$ & \\
& 2.399 & 3.983 & 3.818 & 3.689 & $2.88^{a}$ & $3.681^{a}$ & $3.719^{a}$ & $3.553^{a}$ & $2.92^{b}, 2.72^{c}$ \\
\hline
\end{tabular}

${ }^{a}$ Ref. [33], GGA-FP-LAPW; ${ }^{b}$ Ref. [34] VCA; ${ }^{c}$ Ref. [35] Exp

The present results for $\mathrm{Cd}$ compounds and their ternary alloys are listed in Table VI. It is clearly seen that the values of the refractive index obtained by FP-LMTO method are in reasonable agreement with other results. Figure 8 notes that when $x$ increases, $n$ decreases as well. The refractive indices are related to their energy bands. There is a correlation between these two fundamental properties which has a significant impact on the band structure of semiconductors [44]. Given the fact that the $E_{\mathrm{g}}$ of the material of interest decreases with increasing $x$ (see Table II) and $n$ increases with $x$ (see Table V). The calculated refractive indices versus concentration using FP-LMTO were fitted by a polynomial equation to find $n$ equal to 0.38 . Hence, the refractive index of the alloy depends strongly with concentration $x$ The results are summarized as follows:

$$
\begin{aligned}
& n_{1}(x)=1.96-0.1 x+0.38 x^{2}(\mathrm{FP}-\mathrm{LMTO}) \\
& n_{2}(x)=3.19+0.61 x+0.21 x^{2} \text { (Moss) } \\
& n_{3}(x)=3.44+0.44 x-0.05 x^{2} \text { (Ravindra et al.), } \\
& n_{4}(x)=3.22+0.49 x-0.01 x^{2} \text { (Vandamme) }
\end{aligned}
$$

\subsection{Thermodynamic properties}

In order to study the phase stability of $\mathrm{CdS}_{1-x} \mathrm{Te}_{x}$ alloy, the Gibbs free energy of mixing $\Delta G_{\mathrm{m}}(x, T)$ is calculated in order to access the $T-x$ phase diagram and obtain the critical temperature, $T_{\mathrm{c}}$, for miscibility. More details of the calculations are given in Refs. [45-47]. The Gibbs free energy of mixing, $\Delta G_{\mathrm{m}}$, for alloys can be expressed by

$$
\begin{aligned}
& \Delta G_{\mathrm{m}}=\Delta H_{\mathrm{m}}-T \Delta S_{\mathrm{m}}, \\
& \Delta H_{\mathrm{m}}=\Omega x(1-x), \\
& \Delta S_{\mathrm{m}}=-R[x \ln x+(1-x) \ln (1-x)] .
\end{aligned}
$$

$\Delta H_{\mathrm{m}}$ and $\Delta S_{\mathrm{m}}$ are the enthalpy and entropy of mixing, respectively, $\Omega$ is the interaction parameter and depends on material, $R$ is the perfect gas constant, and $T$ is the absolute temperature. The mixing enthalpy of alloys can be obtained from the calculated total energies as

$$
\Delta H_{\mathrm{m}}=E_{\mathrm{AB} 1-x \mathrm{C} x}-(1-x) E_{\mathrm{AB}}-x E_{\mathrm{AC}},
$$

where $E_{\mathrm{AB} 1-x \mathrm{C} x}, E_{\mathrm{AB}}$, and $E_{\mathrm{AC}}$ are the respective energies of $\mathrm{AB}_{1-x} \mathrm{C}_{x}$ alloy and the binary compounds $\mathrm{AB}$, and $\mathrm{AC}$. We then calculated $\Delta H_{\mathrm{m}}$ to obtain $\Omega$ as a function of concentration. The interaction parameter in- 
creases almost linearly with increasing $x$. From a linear fit we obtained

$$
\mathrm{CdS}_{1-x} \mathrm{Te}_{x} \longrightarrow \Omega[\mathrm{kcal} / \mathrm{mol}]=18.58-9.44 x .
$$

The average values of the $x$-dependent $(\Omega)$ in the range $0<x<1$ derived from these equations for $\mathrm{CdS}_{1-x} \mathrm{Te}_{x}$ alloy is about $13.86 \mathrm{kcal} / \mathrm{mol}$. The larger enthalpy for $\mathrm{CdS}_{1-x} \mathrm{Te}_{x}$ alloy suggests a large value of $\Omega$ and, hence a higher critical temperature. By calculating the temperature composition, phase diagram shows the stable, metastable and unstable mixing regions of the alloy. At a temperature lower than the critical temperature $T_{\mathrm{c}}$, the two binodal points are determined as those points at which the common tangent line touches the $\Delta G_{\mathrm{m}}$ curves. The two spinodal points are determined as those points at which the second derivative of $\Delta G_{\mathrm{m}}$ is zero. The spinodal and binodal curves of the alloys are sketched in Fig. 9.

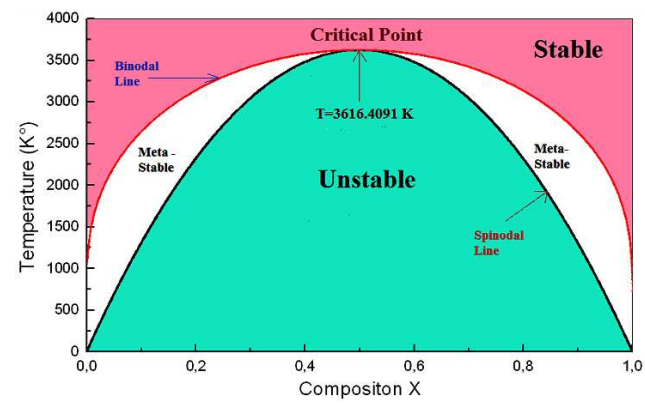

Fig. 9. T-x phase diagram for $\mathrm{CdS}_{1-x} \mathrm{Te}_{x}$ alloy. Black line: binodal curve, red line: spinodal curve.

We have thus calculated the phase diagram using the average values of the $x$-dependent $\Omega$, hence the phase diagram looks symmetric. In Fig. 8, we observed a critical temperature $T_{\mathrm{c}}$ of $3616.4091 \mathrm{~K}$. The spinodal curve in the phase diagram marks the equilibrium solubility limit, i.e. the miscibility gap. A homogeneous alloy is predicted for temperatures and compositions above this curve. The wide range between spinodal and binodal curves indicates that the alloy may exist as a metastable phase. Finally, our results indicate that the $\mathrm{CdS}_{1-x} \mathrm{Te}_{x}$ alloy is stable at high temperature.

\section{Conclusion}

A theoretical study of structural, electronic, optical, and thermodynamic ternary $\mathrm{CdS}_{1-x} \mathrm{Te}_{x}$ is investigated by ab initio FP-LMTO. The agreement between our results and the available experimental theoretical data is considered satisfactory. We studied the composition dependence of the lattice constant, bulk modulus, band gap, refractive index and dielectric function. A small deviation of the lattice constant of the Vegard law was observed in the ternary $\mathrm{CdS}_{1-x} \mathrm{Te}_{x}$. FP-LMTO method is used to calculate refractive index as a function of composition $x$. Our results showed that the refractive index varies linearly compared to other models. The investigation of the thermodynamic stability allowed us to calculate the critical temperatures $\mathrm{CdS}_{1-x} \mathrm{Te}_{x}$ which is of order $3616.4091 \mathrm{~K}$.

\section{Acknowledgments}

Y.A. would like to acknowlegde University Malaysia Perlis for grant No. 9007-00062 and TWAS-Italy for the full support of his visit to JUST-Jordan under TWASUNESCO Associateship.

\section{References}

[1] C.E. Hurwitz, Appl. Phys. Lett. 8, 121 (1966).

[2] F.H. Nicoll, Appl. Phys. Lett. 9, 13 (1966).

[3] S. Wei, S.B. Zhang, Phys. Rev. B 62, 6944 (2000).

[4] N. Benkhettou, D. Rached, B. Soudini, M. Driz, Phys. Status Solidi B 241, 101 (2004).

[5] Y. Al-Douri, Mater. Chem. Phys. 78, 625 (2003).

[6] M. Cote, O. Zakharov, A. Rubio, M.L. Cohen, Phys. Rev. B 55, 13025 (1997).

[7] O. Zakharov, A. Rubio, X. Blase, M.L. Cohen, S.G. Loui, Phys. Rev. B 50, 10780 (1994).

[8] Y. Al-Douri, Mater. Chem. Phys. 82, 49 (2003).

[9] Y. Al-Douri, Ali H. Reshak, H. Baaziz, Z. Charifi, R. Khenata, S. Ahmad, U. Hashim, Solar Energy 84, 1979 (2010).

[10] A. Tomasulo, M.V. Ramakrishna, J. Chem. Phys. 105, 3612 (1996).

[11] M.B. Kanoun, W. Sekkal, H. Aourag, G. Merad, Phys. Lett. A 272, 113 (2000); A. Mujica, A. Rubio, A. Munoz, R.J. Needs, Rev. Mod. Phys. $\mathbf{7 5}$, 863 (2003).

[12] A.E. Merada, M.B. Kanoun, G. Merad, J. Cibert, H. Aourag, Mater. Chem. Phys. 92, 333 (2005).

[13] Y. Al-Douri, H. Baaziz, Z. Charifi, R. Khenata, U. Hashim, M. Al-Jassim, Renewable Energy 45, 232 (2012).

[14] A.S.Z. Lahewil, Y. Al-Douri, U. Hashim, N.M. Ahmed, Solar Energy 86, 3234 (2012).

[15] E. Deligoz, K. Colakoglu, Y. Ciftci, Physica B 373, 124 (2006).

[16] J.P. Perdew, Y. Wang, Phys. Rev. B 45, 13244 (1992).

[17] D.W. Lane, Solar En. Mater. Solar Cells 90, 1169 (2006).

[18] K. Wei, F.H. Pollak, J.L. Freeouf, D. Shvydka, A.D. Compaan, J. Appl. Phys. 85, 7418 (1999).

[19] D.A. Wood, K.D. Rogers, D.W. Lane, J.A. Coath, J. Phys., Condens. Matter 12, 4433 (2000).

[20] S. Ghemid, S. Oundadji, H. Meradji, S. Drablia, S. Labidi, Phys. Proc. 2, 881 (2009).

[21] G.P. Srivastava, G.L. Martins, A. Zunger, Phys. Rev. B 31, 2561 (1985); J.E. Bernard, A. Zunger, Phys. Rev. B 34, 5992 (1986).

[22] S.Y. Savrasov, Phys. Rev. B 54, 16470 (1996).

[23] S. Savrasov, D. Savrasov, Phys. Rev. B 46, 12181 (1992).

[24] D. Rached, M. Rabah, N. Benkhettou, M. Driz, B. Soudini, Physica B 337, 394 (2003).

[25] J.P. Perdew, S. Burke, M. Ernzerhof, Phys. Rev. Lett. 77, 3865 (1996). 
[26] P. Blochl, O. Jepsen, O.K. Andersen, Phys. Rev. B 49, 16223 (1994).

[27] F.D. Murnaghan, Proc. Natl. Acad. Sci. USA 30, 244 (1944).

[28] O. Madelung, M. Scholz, H. Weiss, in: Numerical Data and Functional Relationships in Science and Technology, Eds. Landolt-Bornstein, Vol. 17, Springer, Berlin 1982.

[29] L. Vegard, Z. Phys. 5, 17 (1921).

[30] J. Heyd, J.E. Peralta, G.E. Scuseria, J. Chem. Phys. 123, 174101 (2005).

[31] S. Zerroug, F. Ali Sahraoui, N. Bouarissa, Eur. Phys. J. B 57, 9 (2007).

[32] N.A. Noora, W. Tahir, F. Aslam, A. Shaukat, Physica B 407, 943 (2012).

[33] S. Ouendadji, S. Ghemid, H. Meradji, F. El Haj Hassan, Comp. Mater. Sci. 48, 206 (2010).

[34] L. Hannachi, N. Bouarissa, Superlatt. Microstruct. 44, 794 (2008).

[35] D.W. Palmer, www.semiconductors.co.uk .

[36] S.N. Rashkeev, W.R.L. Lambrecht, Phys. Rev. B 63, 165212 (2001).

[37] W. Sargent, Table of Periodic Properties of the Elements, Sargent-Welch Scientific, Skokie, IL 1980.
[38] M. Alouani, J.M. Koch, M.A. Khan, J. Phys. F 16, 437 (1986).

[39] C. Koenig, M.A. Khan, Phys. Rev. B 17, 6129 (1983).

[40] M.A. Khan, A. Kashyap, A.K. Solanki, T. Nautiyal, S. Auluck, Phys. Rev. B 48, 16947 (1993).

[41] V.P. Gupta, N.M. Ravindra, Phys. Status Solidi B 10, 715 (1980).

[42] N.M. Ravindra, S. Auluck, V.K. Srivastava, Phys. Status Solidi B 93, 155 (1979).

[43] J.P.L. Herve, L.K.J. Vandamme, Infrared Phys. Technol. 35, 609 (1994).

[44] N.M. Ravindra, P. Ganapathy, J. Choi, Infrared Phys. Technol. 50, 21 (2007).

[45] R.A. Swalin, Thermodynamics of Solids, Wiley, New York 1961.

[46] L.G. Ferreira, S.H. Wei, J.E. Bernard, A. Zunger, Phys. Rev. B 40, 3197 (1999).

[47] L.K. Teles, J. Furthmuller, L.M.R. Scolfaro, J.R. Leite, F. Bechstedt, Phys. Rev. B 62, 2475 (2000). 